\title{
Targeting ER stress-induced autophagy overcomes BRAF inhibitor resistance in melanoma
}

\begin{abstract}
Xiao-Hong Ma, ${ }^{1}$ Sheng-Fu Piao, ${ }^{1}$ Souvik Dey, ${ }^{2}$ Quentin Mcafee, ${ }^{1}$ Giorgos Karakousis, ${ }^{3}$ Jessie Villanueva, ${ }^{4}$ Lori S. Hart, ${ }^{2}$ Samuel Levi, ${ }^{5}$ Janice Hu, ${ }^{1}$ Gao Zhang, ${ }^{4}$ Rossitza Lazova, ${ }^{6}$ Vincent Klump, ${ }^{6}$ John M. Pawelek, ${ }^{6}$ Xiaowei Xu, ${ }^{7}$ Wei Xu, ${ }^{1}$ Lynn M. Schuchter, ${ }^{1,8}$ Michael A. Davies, ${ }^{9}$ Meenhard Herlyn, ${ }^{4}$ Jeffrey Winkler, ${ }^{5,8}$ Constantinos Koumenis, ${ }^{2,8}$ and Ravi K. Amaravadi1,8

${ }^{1}$ Department of Medicine, ${ }^{2}$ Department of Radiation Oncology, and ${ }^{3}$ Department of Surgery, University of Pennsylvania, Philadelphia, Pennsylvania, USA. ${ }^{4}$ Molecular and Cellular Oncogenesis Program, Wistar Institute, Philadelphia, Pennsylvania, USA. ${ }^{5}$ Department of Chemistry, University of Pennsylvania, Philadelphia, Pennsylvania, USA. ${ }^{6}$ Department of Dermatology, Yale University, New Haven, Connecticut, USA. ${ }^{7}$ Department of Pathology and Laboratory Medicine and ${ }^{8} \mathrm{Abramson}$ Cancer Center, University of Pennsylvania, Philadelphia, Pennsylvania, USA. 9MD Anderson Cancer Center, University of Texas, Houston, Texas, USA.
\end{abstract}

\begin{abstract}
Melanomas that result from mutations in the gene encoding BRAF often become resistant to BRAF inhibition (BRAFi), with multiple mechanisms contributing to resistance. While therapy-induced autophagy promotes resistance to a number of therapies, especially those that target $\mathrm{PI} 3 \mathrm{~K} / \mathrm{mTOR}$ signaling, its role as an adaptive resistance mechanism to BRAFi is not well characterized. Using tumor biopsies from $\mathrm{BRAF}^{\mathrm{V} 600 \mathrm{E}}$ melanoma patients treated either with BRAFi or with combined BRAF and MEK inhibition, we found that BRAFi-resistant tumors had increased levels of autophagy compared with baseline. Patients with higher levels of therapyinduced autophagy had drastically lower response rates to BRAFi and a shorter duration of progression-free survival. In BRAF ${ }^{\mathrm{6} 00 \mathrm{E}}$ melanoma cell lines, BRAFi or BRAF/MEK inhibition induced cytoprotective autophagy, and autophagy inhibition enhanced BRAFi-induced cell death. Shortly after BRAF inhibitor treatment in melanoma cell lines, mutant BRAF bound the ER stress gatekeeper GRP78, which rapidly expanded the ER. Disassociation of GRP78 from the PKR-like ER-kinase (PERK) promoted a PERK-dependent ER stress response that subsequently activated cytoprotective autophagy. Combined BRAF and autophagy inhibition promoted tumor regression in BRAFi-resistant xenografts. These data identify a molecular pathway for drug resistance connecting BRAFi, the ER stress response, and autophagy and provide a rationale for combination approaches targeting this resistance pathway.
\end{abstract}

\section{Introduction}

The 50\% response rate and 7 -month progression-free survival (PFS) observed in clinical trials of BRAF inhibition (BRAFi) using the inhibitors vemurafenib (also known as PLX4032) and dabrafenib represents a major advance in the treatment of melanoma patients harboring the BRAF ${ }^{\mathrm{V} 600 \mathrm{E}}$ mutation. However, nearly $100 \%$ of patients eventually progress on BRAFi therapy (1-3). Recently, a number of resistance mechanisms to BRAF inhibitors were proposed, involving either reactivation of the MAPK kinase pathway or concurrent activation of alternative growth factor signaling pathways (4). These studies demonstrate that BRAFi resistance is likely to be heterogeneous, encompassing a spectrum of molecular changes that are being found in biopsies obtained during treatment and at the time of progression.

Drug-induced autophagy has emerged as a common pathway of resistance to a number of kinase inhibitors. In most of these cases, the focus of the effects of kinase inhibitors on autophagy has been on the well-characterized link between PI3K/AKT/mTOR signal-

Authorship note: Xiao-Hong Ma and Sheng-Fu Piao contributed equally to this work.

Conflict of interest: Ravi K. Amaravadi and Jeffrey Winkler are coinventors of a licensed patent, which has generated no revenue, protecting the structure and use of Lys05 and derivatives.

Citation for this article: J Clin Invest. 2014;124(3):1406-1417. doi:10.1172/JCI70454 ing and autophagy (5). Much less is known about the link between MAPK signaling and autophagy. Autophagy is a multistep process that involves the sequestration of organelles and proteins in autophagic vesicles (AVs) and subsequent lysosome-dependent degradation of these contents, leading to recycling of macromolecules to fuel further growth. There is a large body of evidence to indicate that autophagy can promote cell survival within the tumor microenvironment $(5,6)$, depending on cellular and tissue context. Within the same cell, cytoprotective autophagy is often upregulated early during cancer therapy and concurrently with cell death pathways such as apoptosis. Especially in vivo, therapyinduced autophagy that is observed within intact tumor cells in a shrinking tumor may be indicative of cells that are not destined to die, but are adapting to therapeutic stress and will eventually give rise to recurrent tumors. Based on reports that autophagy inhibition can augment the antitumor efficacy of therapies that induce autophagy (7-10), numerous clinical trials involving the firstgeneration autophagy inhibitor hydroxychloroquine (HCQ) have been launched $(5,11)$. We previously reported that high levels of autophagy prior to treatment predict invasiveness, poor response to cytotoxic chemotherapy, and shortened survival in metastatic melanoma (12). Elevated levels of autophagy in primary tumors also correlated with proliferation and lymph node metastases in human melanoma tumors $(13,14)$. These results demonstrate that autophagy levels can be high before treatment in melanoma, but 
A



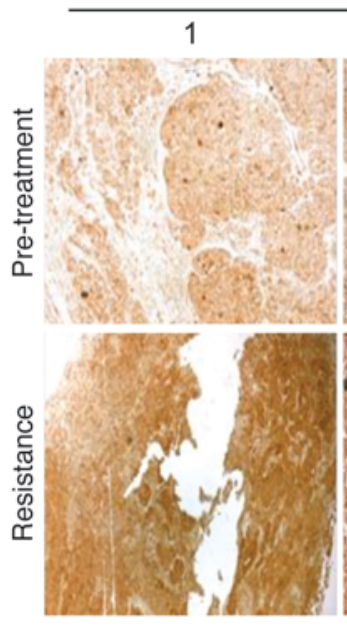

Patient

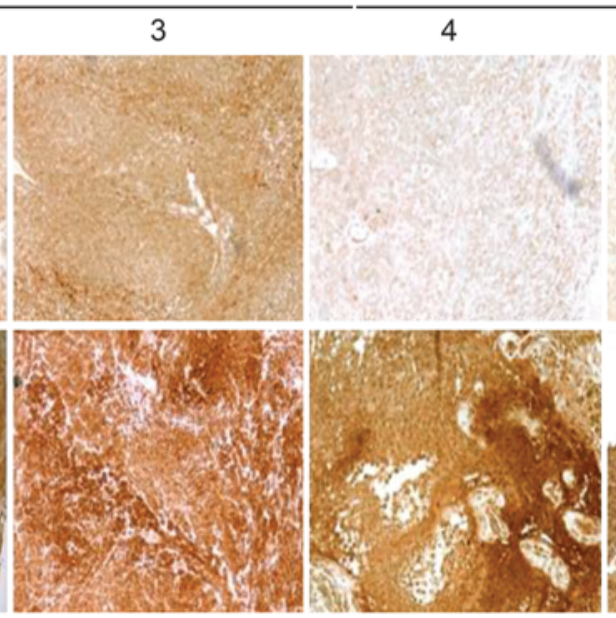

Res=Pre

$(2 / 15)$

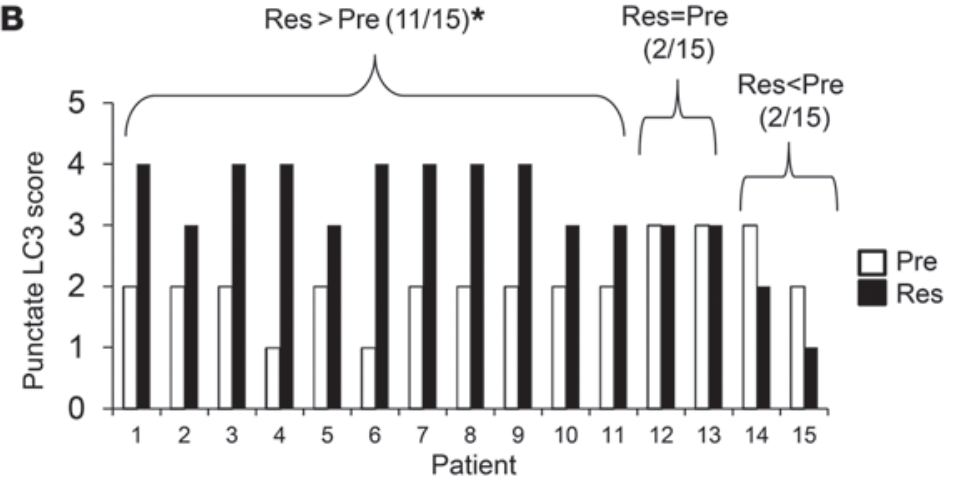

C

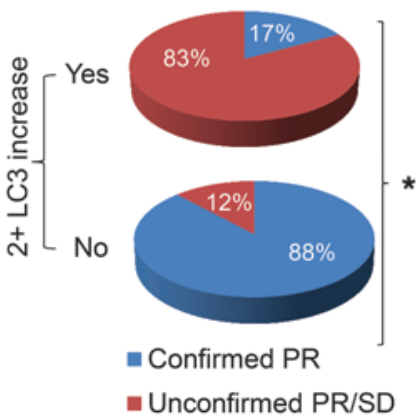

Figure 1

BRAFi induces autophagy in the tumors of BRAF mutant melanoma patients. (A) Representative images of pretreatment and resistance samples from patients treated with the BRAF inhibitor vemurafenib. IHC staining (brown) was conducted for the autophagy marker LC3. Original magnification, $\times 10$. (B) IHC scores for pretreatment (Pre) and resistance (Res) samples for 15 patients. ${ }^{*}<0.05$, Res $>$ Pre vs. Res $=$ Pre and Res $<$ Pre. (C) RECIST responses based on a 2+ increase in LC3B staining in resistance versus pretreatment samples. PR, partial response; SD, stable disease. ${ }^{*} P<0$ 0.05. (D) PDF in BRAF mutant melanoma patients based on LC3B IHC staining score. $P=0.0487$, log-rank test.

may be induced even further during therapies, and support the notion that autophagy modulation may be especially effective as a therapeutic strategy in melanoma. To date, the role of autophagy as a potential druggable adaptive resistance mechanism in the setting of BRAFi has not been investigated.

Here we report elevated levels of autophagy after treatment with vemurafenib, dabrafenib, or the combination of dabrafenib and the MEK inhibitor trametinib in melanoma patient tumor samples that persist at the time of progression. The mechanistic link between BRAF signaling and autophagy, and the effects of autophagy inhibition in BRAFi-sensitive and BRAFi-resistant cell lines, were studied. Our findings indicate that BRAFi induces cytoprotective autophagy through activation of an ER stress response, and that targeting multiple components of the ER stress-autophagy pathway can overcome BRAFi resistance in melanoma.

\section{Results}

Vemurafenib-induced autophagy in patient tumor samples. To determine whether autophagy levels increase in response to BRAFi, paired biopsy samples from 15 patients with BRAF mutant melanoma treated with BRAF inhibitors were stained for the autophagy marker LC3B. LC3 is an ubiquitin-like cytoplasmic protein that is conjugated to the surface of AVs as they are formed. Autophagy levels are directly reflected by the degree of punctuate LC3B (14). The clinical characteristics of the 15 advanced BRAF mutant melanoma patients included in our analysis are presented in Supplemental Table 1 (supplemental material available online with this article; doi:10.1172/JCI70454DS1). Patients were all treated at therapeutic doses with the single-agent BRAF inhibitors vemurafenib $(n=10)$ or dabrafenib $(n=2)$ or with the combination of the BRAF inhibitor dabrafenib and the MEK inhibitor trametinib $(n=3)$. For each of the 15 patients, we applied our previously validated LC3B immunohistochemistry (IHC) assay (14) to tissue biopsies from tumors prior to treatment (pretreatment samples) and to tumors that were regrowing despite treatment with BRAF inhibitors (resistance samples) (Figure 1A). The LC3B IHC scores for pretreatment versus resistance samples were $13 \%$ versus $7 \%$ for $1+, 67 \%$ versus $7 \%(P<0.05)$ for $2+, 20 \%$ versus $40 \%(P<0.05)$ for $3+$, and $0 \%$ versus $46 \%(P<0.05)$ for $4+$. LC3B scores in pretreatment samples were lower than those in resistance samples in $74 \%$ of cases, equal in $13 \%$ of cases, and higher in $13 \%$ of cases (Figure 1B). Higher-powered views of LC3B staining in pretreatment and 
A

BRAFi-sensitive (nanomolar $\mathrm{IC}_{50}$ )

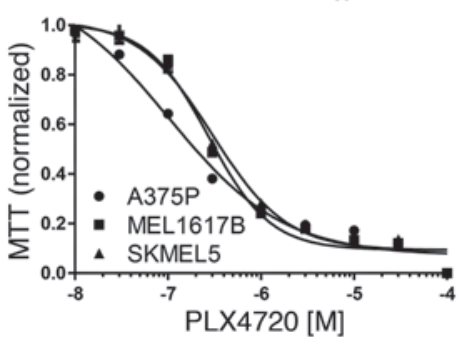

C
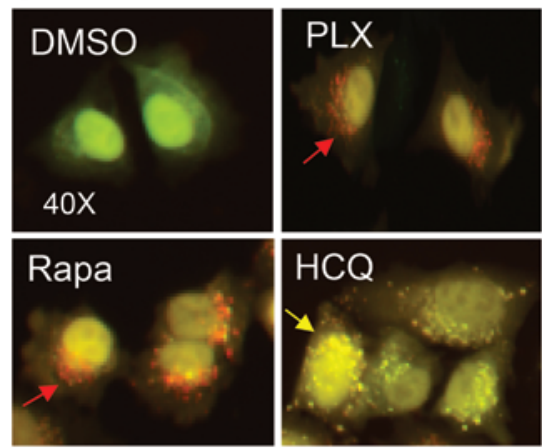

E

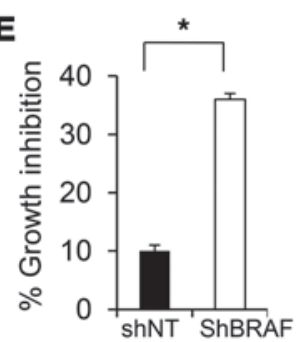

\section{$\mathbf{F}$}

BRAFi-resistant (micro/millimolar $\mathrm{IC}_{50}$ )
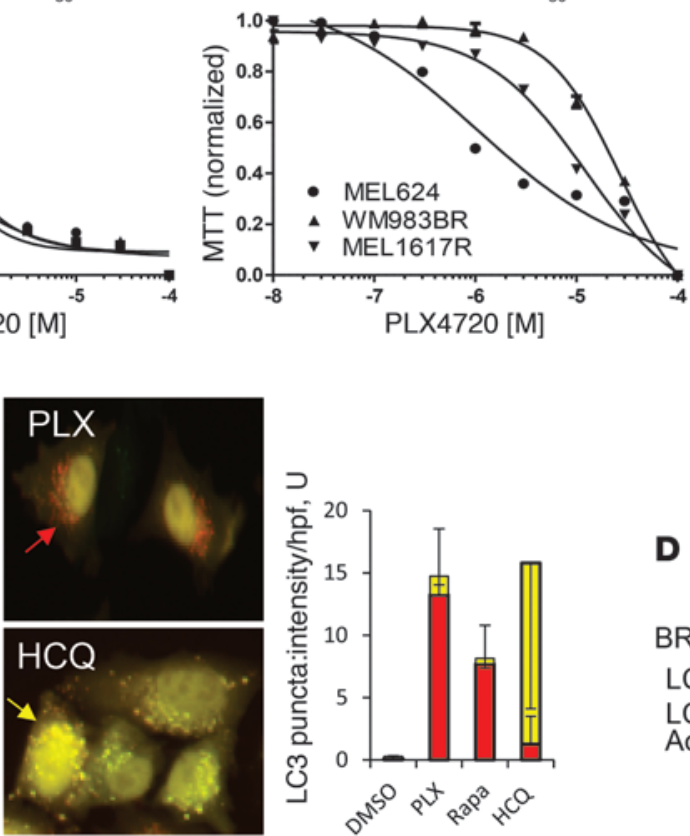
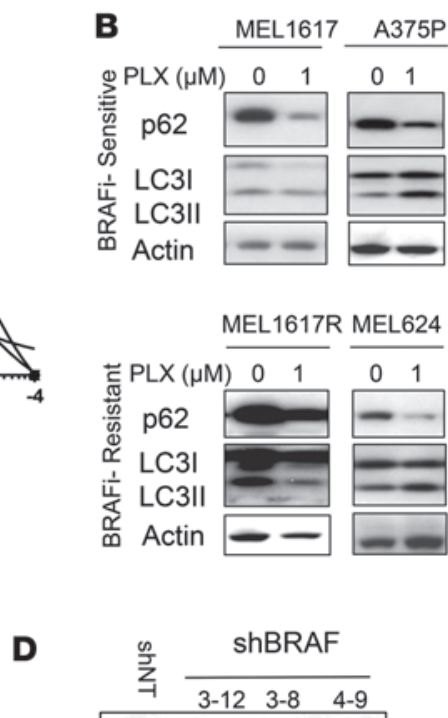
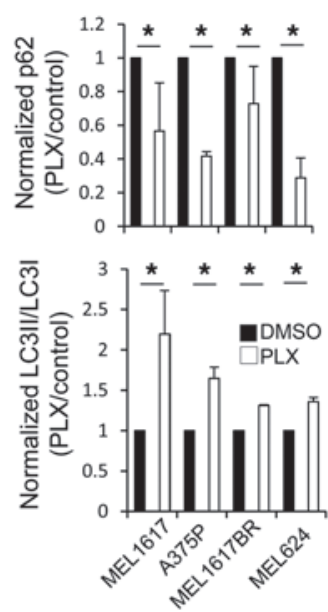
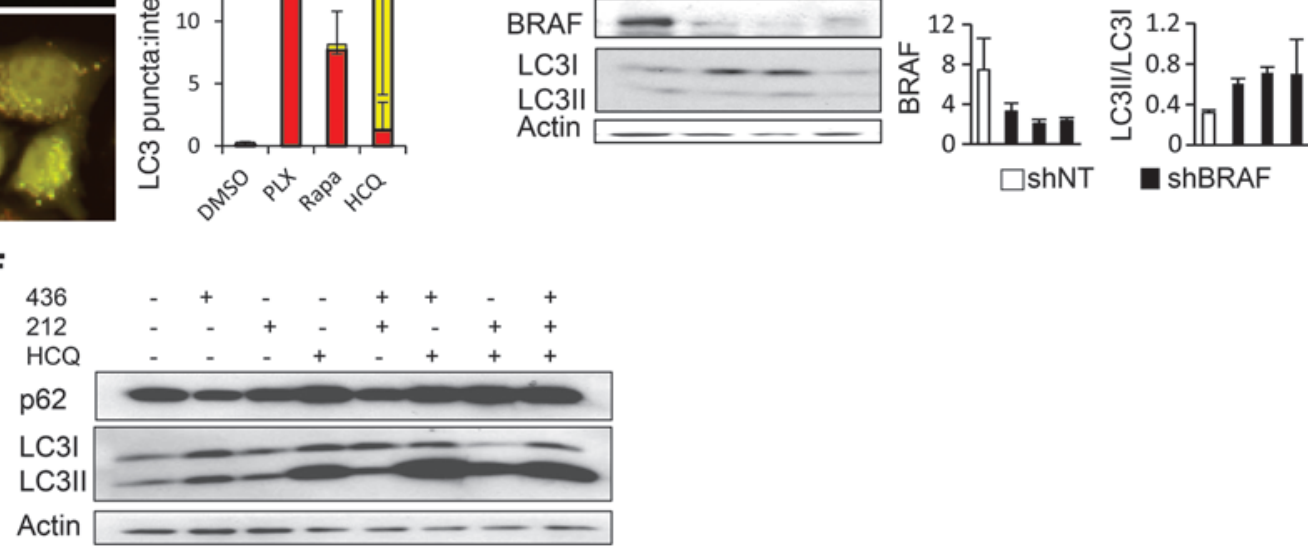

\section{Figure 2}

BRAFi induces cytoprotective autophagy. (A) Dose-dependent growth impairment of 6 BRAF mutant cell lines treated in 6 replicates with PLX4720 (72-hour MTT assay). Results are mean \pm SEM. (B) Immunoblots and gel density quantifications (mean \pm SEM for 3 separate experiments) directed against autophagy markers in 48-hour cell lysates from the indicated cell lines treated with vehicle or PLX4720. (C) A375P mCherry-GFP-LC3 cells were treated with DMSO, $1 \mu \mathrm{M}$ PLX4720, 500 nM rapamycin, or $10 \mu \mathrm{M}$ HCQ. Representative merged images of red and green channels after 8 hours of treatment are shown. Original magnification, $\times 40$. Autophagic flux (measured by red puncta) and distal blockade of autophagy (measured by yellow puncta) was quantified (mean \pm SD of triplicate experiments). (D) Immunoblotting against autophagy markers (mean \pm SEM) in untreated A375PshNT and 3 distinct A375PshBRAF clones. (E) Percent growth inhibition after 48 hours of $10 \mu \mathrm{M} \mathrm{HCQ}$ treatment compared with control in A375PshNT and A375PshBRAF cells (MTT assay). Results are mean \pm SEM. (F) A375P cells were treated with vehicle, the BRAF inhibitor GSK2118436, the MEK inhibitor GSK1120212, $10 \mu \mathrm{M} \mathrm{HCQ}$, or the indicated combinations. Shown are 48-hour immunoblots directed against the indicated proteins. ${ }^{*} P<0.05$.

progression samples showed not only increased total LC3B signaling, but an increase in the punctuate staining pattern that is typically associated with AV buildup (Supplemental Figure 1A). Serial tumor biopsies from 2 patients with BRAF mutant melanoma enrolled on clinical trials of vemurafenib were obtained and analyzed by electron microscopy. Even within 15 days of starting treatment, cells surviving the stress of BRAFi, with intact nuclear and cytoplasmic membranes, had a 2- to 6-fold increase in AVs compared with baseline measurements (Supplemental Figure 1B). Biopsy of tumor tissue at the time of progression demonstrated persistently elevated levels of autophagy in both patients. These findings demonstrated that at therapeutic doses of BRAF inhibitors, autophagy is induced early in patient tumors, and elevated autophagy persists at the time of tumor progression. The clinical relevance of the observed increase in autophagy at the time of resistance as a potential adaptive resistance mechanism in melanoma cells was apparent when we correlated changes in LC3B levels and absolute LC3B levels in progression samples with clinical outcomes. Patients who had a $2+$ increase in LC3B staining in progression versus baseline samples had a $17 \%$ confirmed partial response rate (>30\% shrinkage of tumor compared with baseline on 2 computed tomography scans separated by at least 30 days), whereas patients who did not have a $2+$ increase in LC3B staining in progression versus baseline biopsies had an $88 \%$ confirmed partial response rate $(P<0.05$, Fisher exact test; Figure $1 \mathrm{C})$. Similarly, patients with 4+ LC3B staining at progression had significantly 
A
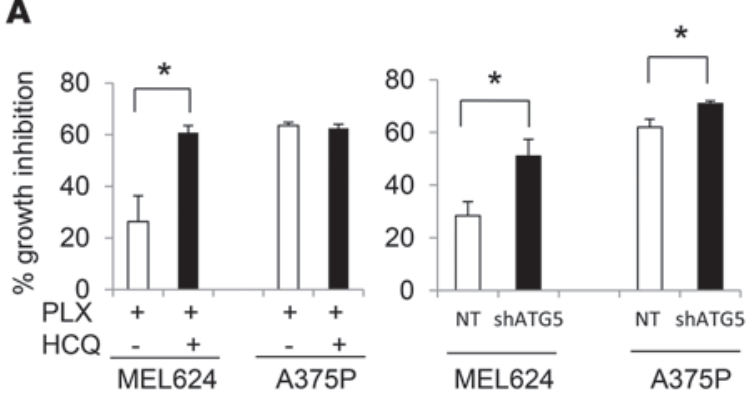

B
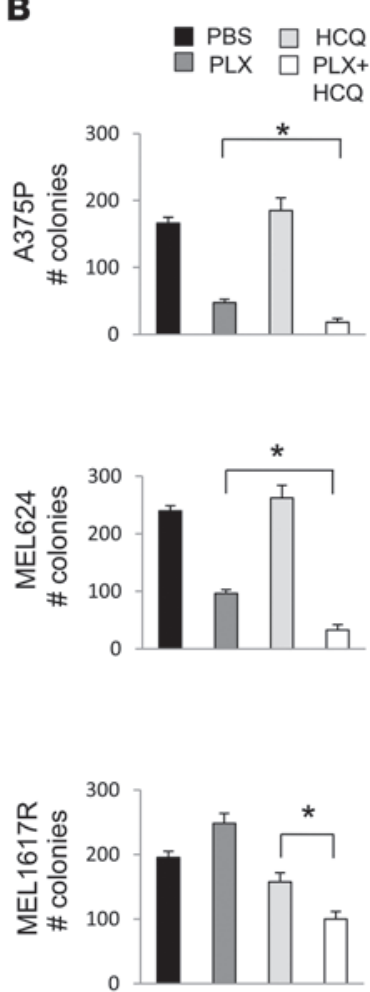
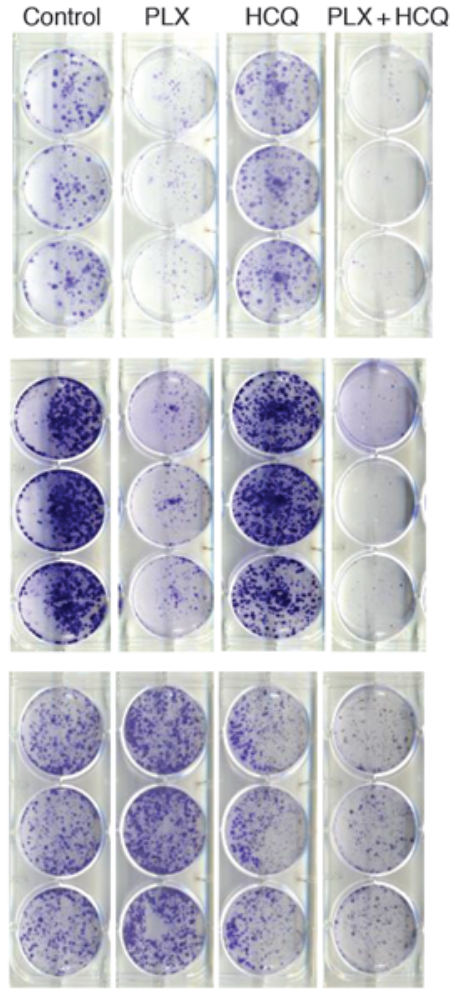

C
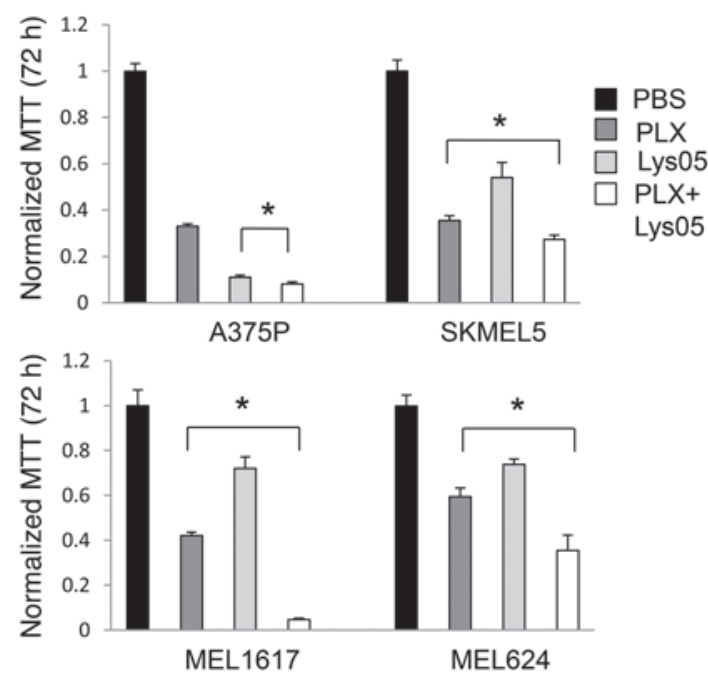

D

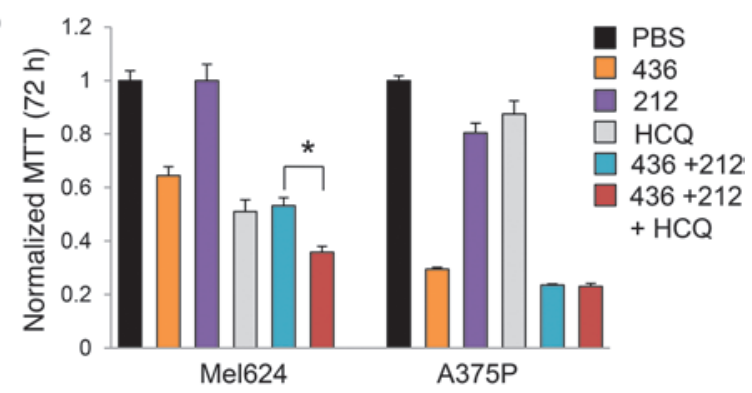

Figure 3

Autophagy inhibition augments growth impairment associated with BRAFi. (A) Percent growth inhibition (mean \pm SEM) compared with control for $1 \mu \mathrm{M}$ PLX4720 treatment with or without $10 \mu \mathrm{M} \mathrm{HCQ}$ (left), or for $1 \mu \mathrm{M}$ PLX4720 treatment of MEL624shNT, MEL624shATG5, A375PshNT, and A375PshATG5 clones (right). (B) 14 day clonogenic growth assays for BRAFi-sensitive A375P and BRAFi-resistant MEL1617R and MEL624. Cells were plated in triplicate and immediately treated with PBS vehicle, $500 \mathrm{nM} \mathrm{PLX} 4720,5 \mu \mathrm{M} \mathrm{HCQ}$, or the combination. Colony counts (mean \pm SEM) are presented. (C) 72-hour MTT assay was conducted after treating the indicated cell lines with PBS, $1 \mu$ M PLX4720, $3 \mu$ M Lys05, or the combination. Results are mean \pm SEM normalized to control. (D) MEL624 and A375P cells were treated with $30 \mathrm{nM}$ GSK2118436, $20 \mathrm{nM}$ GSK1120212, $10 \mu \mathrm{M} \mathrm{HCQ}$, and the indicated combinations. 72-hour MTT (mean \pm SEM) was performed. ${ }^{*} P<0.05$.

shorter PFS (median 229 days) than patients with lower levels of LC3B staining in progression samples (median 275 days; hazard ratio, $0.28 ; 95 \%$ CI, 0.08-0.99; $P=0.0487$, log-rank test; Figure 1D). These clinical data demonstrated that autophagy is a potentially druggable adaptive resistance mechanism to BRAFi.

BRAFi induces autophagy in BRAF mutant melanoma cell lines. To determine the role of autophagy in BRAFi resistance, we assessed autophagy induction in $6 \mathrm{BRAF}^{\mathrm{V} 600 \mathrm{E}}$ melanoma cell lines, including a set of paired cell lines that were BRAFi-sensitive, but had acquired BRAFi resistance through chronic in vitro exposure (15). We categorized cell lines as either BRAFi-sensitive (A375P, SKMEL5, MEL1617) or BRAFi-resistant (MEL1617R, WM983BR,
MEL624) by treating cells with the highly specific BRAF inhibitor PLX4720 and identifying the $\mathrm{IC}_{50}$. Based on previous literature for BRAFi resistance mechanisms $(15,16)$, we classified cell lines with nanomolar $\mathrm{IC}_{50} \mathrm{~S}$ as BRAFi-sensitive and those with micromolar or millimolar $\mathrm{IC}_{50}$ S as BRAFi-resistant (Figure $2 \mathrm{~A}$ ). After 48 hours of treatment with $1 \mu \mathrm{M}$ PLX4720, immunoblotting against the autophagy markers LC3 and p62 was conducted. When conjugated to the AV surface, the conjugated form of LC3 (LC3II) migrates separately from the unconjugated, free cytoplasmic form (LC3I). The LC3II/LC3I ratio is reflective of AV accumulation (17). When autophagy is induced, the LC3II/LC3I ratio is increased, and levels of the protein cargo adaptor p62 drop as it gets digested within 
A

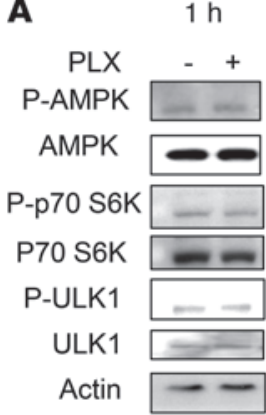

$4 \mathrm{~h}$

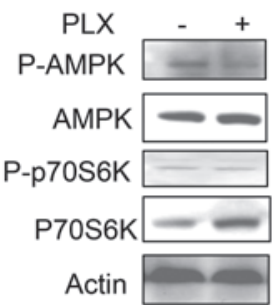

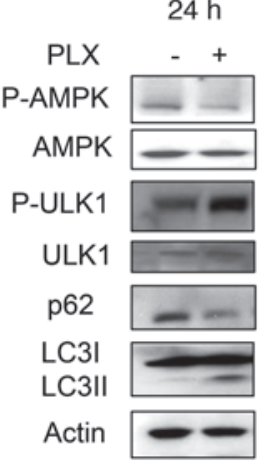

B

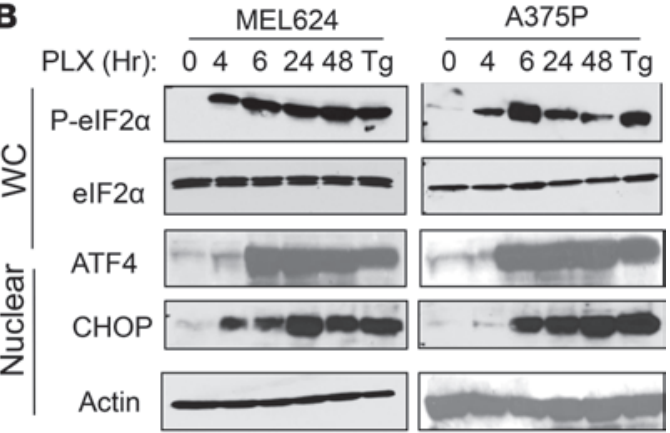

C

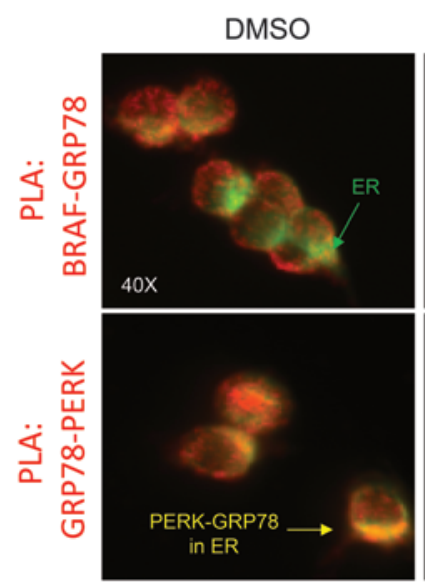

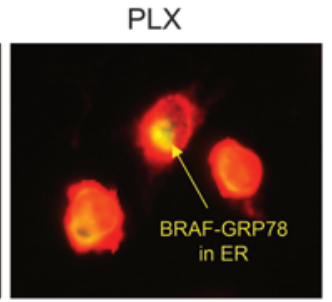

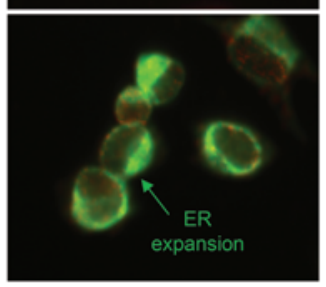

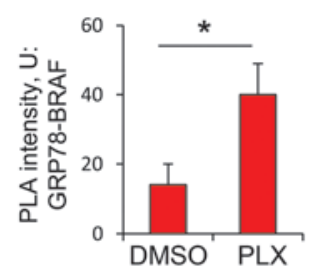

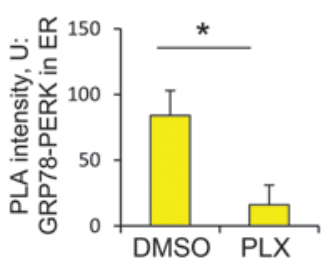

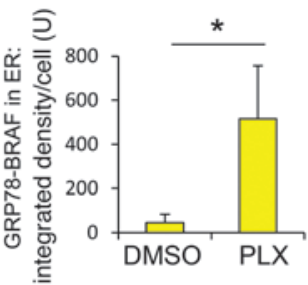

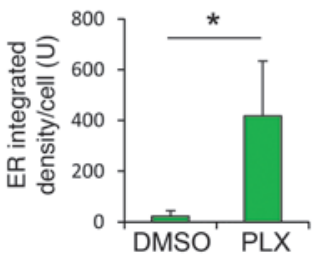

Figure 4

BRAFi induces an early ER stress response. (A) Immunoblotting against the indicated signaling markers after treatment of MEL624 cells with vehicle or $1 \mu \mathrm{M}$ PLX4720 for the indicated times. P-, phospho-. (B) MEL624 and A375P cells were treated with $1 \mu \mathrm{M}$ PLX4720 for the indicated times. Whole-cell and nuclear lysates were subjected to immunoblotting. Tg, thapsigargin. (C) PLA performed on MEL624 cells treated for 30 minutes with DMSO or $10 \mu \mathrm{M}$ PLX4720 and immunofluorescence for the ER resident protein disulfide isomerase (green), which reflects total ER area. Red fluorescence reflects a protein-protein interaction. Original magnification, $\times 40$. Quantification of red, green, and yellow (colocalization) signals (mean $\pm \mathrm{SD}$ ) reflects at least 3 separate experiments.

functional AVs (18). After treatment with $1 \mu \mathrm{M}$ PLX4720, we observed a significant increase in the LC3II/LC3I ratio and a significant decline in p62 in all cell lines (Figure 2B).

To ensure that BRAFi was in fact inducing autophagy and not blocking distal clearance of AVs, A375P mCherry-eGFP-LC3 cells were treated with DMSO, $1 \mu \mathrm{M}$ PLX4720, $500 \mathrm{nM}$ rapamycin, or $10 \mu \mathrm{M}$ HCQ (Figure 2C). In the absence of any treatment, diffuse LC3 fluorescence was observed. Treatment with PLX4720 produced small red puncta, similar to the canonical autophagy inducer rapamycin, indicative of increased production of autophagosomes and increased autophagic flux, since functional fusion with the lysosome allows quenching of the GFP signal. In contrast, treatment with HCQ produced yellow puncta, reflecting lysosomal impairment and distal autophagy blockade producing persistence of green and red fluorescence (18). To ensure that autophagy was induced by BRAFi and not an off-target effect of PLX4720, we used control nontarget shRNA (shNT) and shRNA against BRAF (shBRAF) in BRAFi-sensitive A375P cells, generating the stable clones A375PshNT and A375PshBRAF cells, respectively. Autophagy induction, as evidenced by elevated LC3II/LC3I ratio, was observed in all subclones of A375PshBRAF compared with A375PshNT cells (Figure 2D). Treatment of A375PshNT and
A375PshBRAF cells with HCQ for 48 hours demonstrated that autophagy inhibition produced a more than 3 -fold increase in growth inhibition in A375PshBRAF versus A375PshNT cells (Figure $2 \mathrm{E}$ ), which indicated that autophagy associated with genetic BRAF suppression was cytoprotective.

Finally, we determined the effect on autophagy and cell death of MAPK pathway inhibition with a second BRAF inhibitor, GSK2118436, the MEK inhibitor GSK1120212, and combined BRAFi and MEK inhibition. This BRAF/MEK inhibitor combination is currently showing promising activity and acceptable safety in human clinical trials (19). Treatment with GSK2118436 or GSK1120212 alone clearly induced autophagy, with increased LC3II/LC3I ratio and decreased p62, at the 48-hour time point (Figure 2F). BRAF/MEK inhibition produced an additive increase in LC3II/LC3I ratio, but the combination of GSK2118436 or GSK1120212 with HCQ produced substantial increases in LC3II/LC3I ratio and p62 levels, and the triple drug combination (GSK2118436, GSK1120212, and HCQ) produced the most substantial increase in LC3II/LC3I ratio and buildup of p62 (Figure $2 \mathrm{~F}$ ), indicative of simultaneous autophagy induction and distal autophagy blockade. Similar results were observed in BRAFi-resistant MEL624 cells (Supplemental Figure 2A). 
A

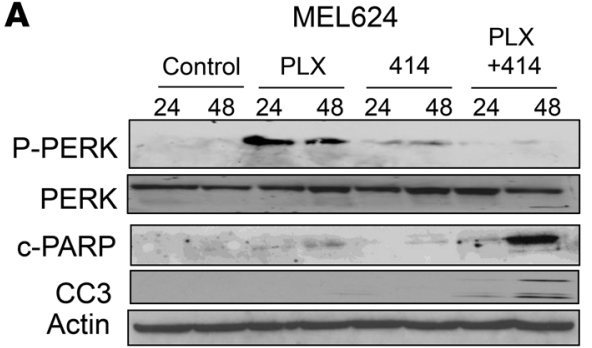

C

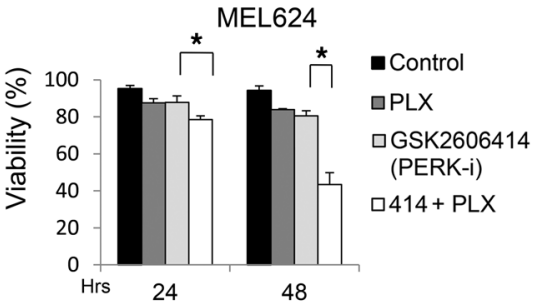

B

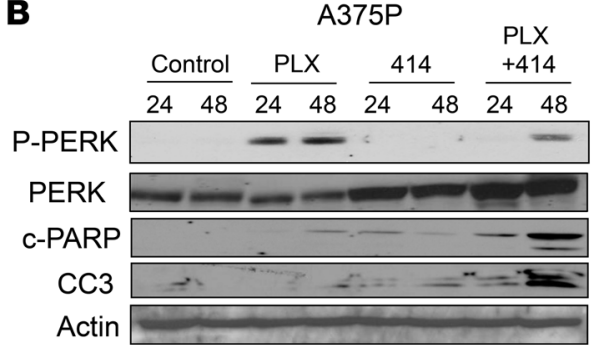

D
$\mathbf{E}$

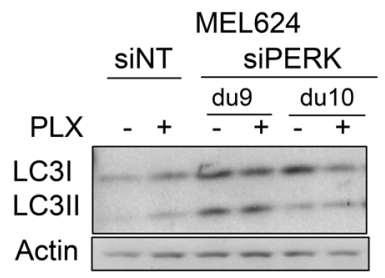

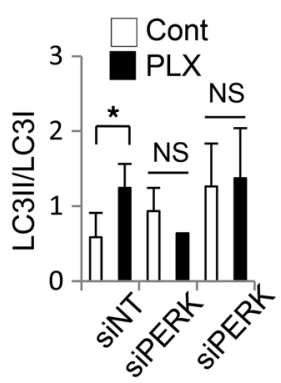

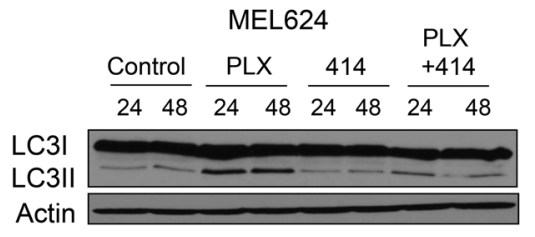

$\mathbf{F}$
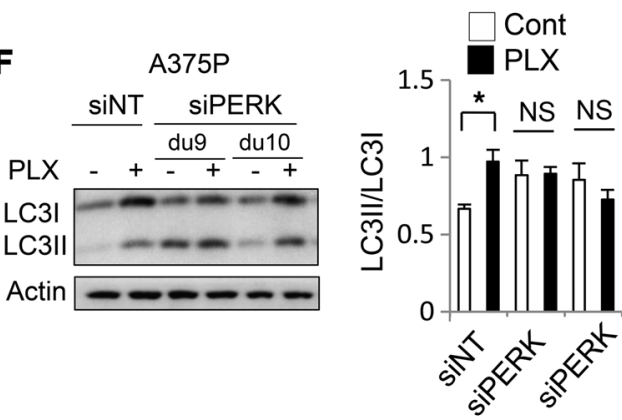

Figure 5

Targeting the BRAFi-induced ER stress response blocks autophagy and enhances cell death. (A and B) The indicated cells were treated with vehicle, $1 \mu \mathrm{M}$ PLX4720, $1 \mu \mathrm{M}$ of the PERK inhibitor GSK2606414, or both for 24 or 48 hours. Immunoblots against the indicated ER stress and apoptosis markers are shown. C-, cleaved; CC3, cleaved caspase-3. (C) Cell viability (Trypan blue staining). Results are mean \pm SEM. ${ }^{\star} P<0.05$. (D) Immunoblot against LC3 in MEL624 cells treated with vehicle, $1 \mu$ M PLX4720, 1 MM GSK2606414, or both for 24 hours. (E and F) MEL624 and A375P cells were treated with PLX4720 (24 hours) and/or siRNA (48 hours) against nontarget control and PERK. Representative immunoblots and LC3II/LC3I ratios (mean \pm SEM; based on gel density quantification from 2 separate experiments) are shown. du, duplex.

Autophagy inbibition augments BRAFi-associated growth impairment. HCQ blocks autophagy by impairing lysosomal function and causing accumulation of AVs with undigested contents (8). Pharmacological inhibition of autophagy with HCQ was compared with genetic inhibition of autophagy using shRNA against ATG5 in BRAFi-resistant MEL624 and BRAFi-sensitive A375P cells (Figure 3A). Stable clones of MEL624shNT, MEL624shATG5, A375PshNT, and A375PshATG5 cells were selected (Supplemental Figure 2B). Despite the finding that HCQ produced an equivalent degree of autophagy inhibition in MEL624 versus A375P cells (Supplemental Figure 2C), addition of HCQ significantly augmented PLX4720-induced growth impairment (as measured by 72-hour MTT assay) nearly 2-fold in MEL624 cells, but not in BRAFi-sensitive A375P cells. Knockdown of ATG5 in MEL624 cells produced a 2 -fold significant augmentation of growth inhibition, nearly identical to the effects of HCQ treatment (Figure 3A). Long-term autophagy suppression augmented PLX4720-associated suppression of colony formation in a 14-day clonogenic assay for both BRAFi-sensitive A375P and BRAFi-resistant MEL1617R and MEL624 cells (Figure 3B). Our previous work demonstrated that autophagy levels in cancer cells grown as $3 \mathrm{D}$ spheroids in a collagen matrix closely resemble autophagy measured in tumor cells within a tumor microenvironment (12). Treatment with $10 \mu \mathrm{M}$ HCQ significantly augmented BRAFi-induced cell death in BRAFi-sensitive A375P, WM983B, and MEL1617 cells as well as in BRAFi-resistant MEL624, MEL1617R, and WM983BR cells grown as 3D spheroids (Supplemental Figure 3). Recently, our group reported the synthesis and characterization of a novel lysosomal autophagy inhibitor, Lys05, which is 10 -fold more potent as an autophagy inhibitor than HCQ (20). PLX4720 in combination with $1 \mu \mathrm{M}$ Lys05 resulted in additive cytotoxicity in A375P, SKMEL5, MEL1617, and MEL624 cells (Figure 3C). Autophagy inhibition with HCQ also augmented the cytotoxicity of the combined BRAF/MEK inhibitors GSK2118436 and GSK1120212, but only in BRAFi-resistant MEL624 cells, not in BRAFi-sensitive A375P cells (Figure 3D). Importantly, there was no antagonism observed when HCQ was combined with BRAF/MEK inhibitors in BRAFi-sensitive cells. Taken together, these findings indicate that BRAFi-induced autophagy is a cytoprotective stress response that is limiting, not promoting, BRAFi-induced cell death. While BRAFi activates autophagy in both BRAFi-sensitive and BRAFiresistant cells, the latter may be more reliant on autophagy as a survival mechanism, and therefore are more reliably sensitive across models to autophagy inhibition. 

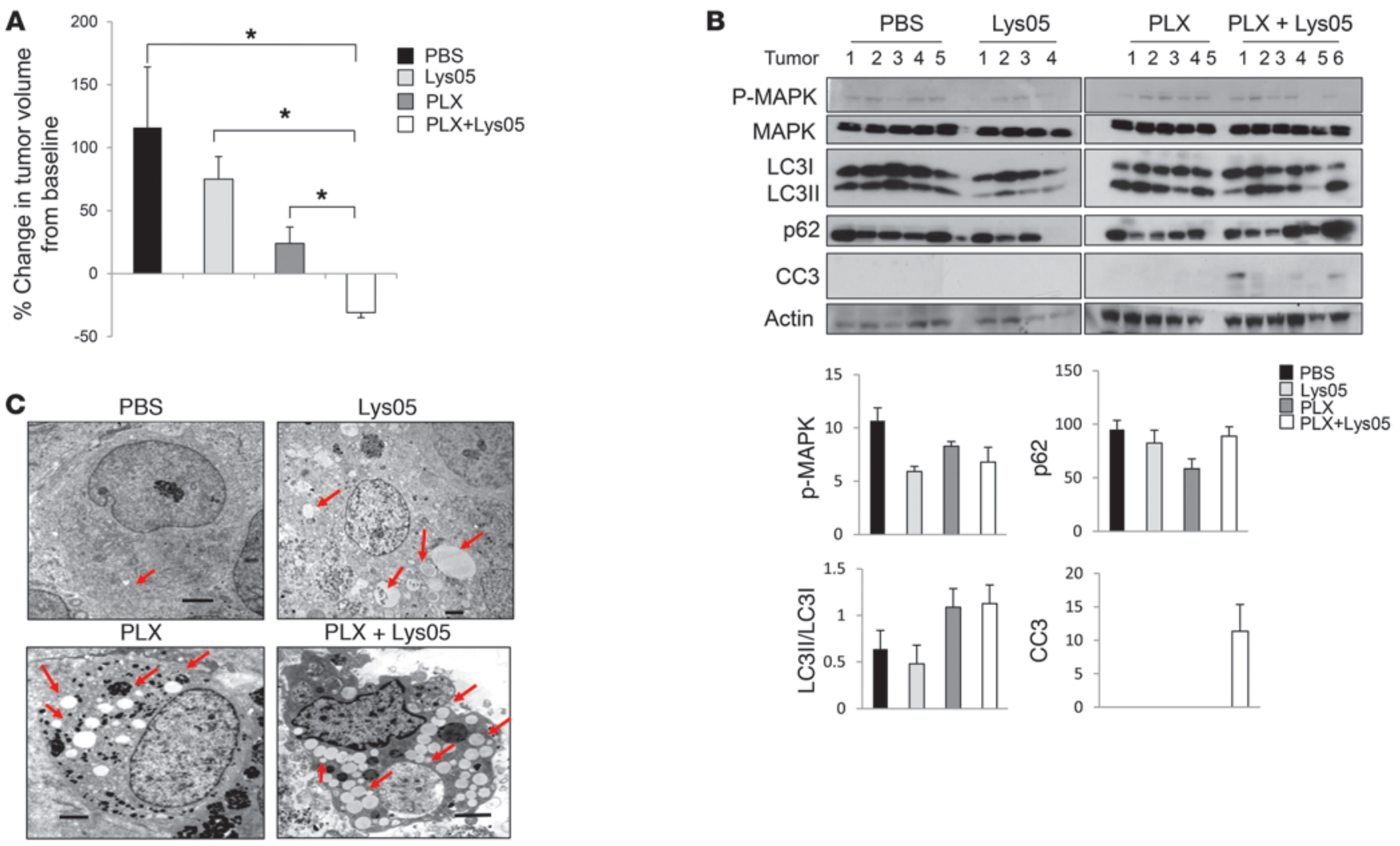

Figure 6

Autophagy inhibition augments BRAFi-induced cell death and antitumor activity in vivo. (A and B) Mice bearing MEL624 melanoma xenografts were treated with PBS or Lys05 (40 mg/kg) i.p. daily and fed either control or PLX4720-infused chow (4-6 per cohort). Tumors were harvested after 22 days of treatment. (A) Change in tumor volume from baseline. Results are mean \pm SEM. ${ }^{*} P<0.05$. (B) Immunoblots and gel quantification (mean \pm SEM) of individual tumors. (C) Representative electron micrographs. Red arrows denote AVs. Scale bars: $2 \mu \mathrm{m}$.

PLX4720-induced autophagy is associated with early ER stress. Next, we investigated the mechanism by which PLX4720 induces autophagy. Autophagy can be activated by alterations in various growth factor signaling cascades (5). The major known direct signaling regulators of autophagy include mammalian target of rapamycin complex 1 (mTORC1), AMPK, and specific components of the ER stress response (5). Previous studies suggest that BRAFi can potentially activate AMPK by disinhibiting liver kinase B1 (LKB1) $(21,22)$. Activated AMPK can phosphorylate and activate the unc-51-like kinase 1 (ULK1) complex, one of the first complexes involved in initiating autophagy (23). Activated mTORC1 phosphorylates and inhibits multiple components of the ULK1 complex. Inhibition of mTORC1 signaling leads to dephosphorylation of the inhibitory phosphorylation site Ser757 on ULK1 (24). Within 1 and 4 hours of BRAF inhibitor treatment, no significant change was observed in the levels of phospho-AMPK (Thr172; indicating activation) or phospho-p70 S6 kinase (phospho-p70S6K) (Thr308; indicating activation) upstream of mTORC1 in the BRAFi-resistant MEL624 cells (Figure 4A). By 24 hours, when there was clear evidence of PLX4720-induced autophagy (increased LC3II/LC3I ratio and decreased p62), there was a modest decline in phospho-AMPK levels, indicative of decreased AMPK activity. Phospho-ULK1 (Ser757) was increased (Figure 4A), reflecting decreased ULK1 activity. These results indicate that major changes in mTORC1, AMPK, or ULK1 signaling are not associated with BRAFi-induced autophagy.
In contrast, multiple markers of the ER stress response were elevated at early time points in response to BRAFi. The ER stress response can be activated by a number of cellular stresses and is controlled primarily by the gatekeeper GRP78, which binds and limits activation of 3 transmembrane proteins: PKR-like ERkinase (PERK), inositol-requiring kinase $1 \alpha$ (IRE1 $\alpha)$, and activating transcription factor $6 \alpha$ (ATF6 $\alpha$ ) (reviewed in refs. 25, 26). The most well-studied connection between the ER stress response and autophagy involves the PERK arm of the ER stress response. PERK activation leads to phosphorylation of eukaryotic initiation factor $2 \alpha(\mathrm{eIF} 2 \alpha)$ and increased expression of the transcription factor activating transcription factor 4 (ATF4) and C/EBP homologous protein (CHOP) (26). Immunoblotting against whole-cell lysates indicated striking upregulation of ATF4 within 4 hours in MEL624 and A375P cells treated with $10 \mu \mathrm{M}$ PLX4720, but not the $1 \mu \mathrm{M}$ lower dose (Supplemental Figure 4A). Dose-dependent decreases in p62 levels, indicative of autophagy induction, were most evident at 6 hours, which indicates that autophagy closely followed induction of the ER stress response. However, within 6 hours of low-dose PLX4720 treatment $(1 \mu \mathrm{M})$, substantial increases in the levels of phospho-eIF2 $\alpha$ were observed in BRAFi-sensitive A375P and BRAFi-resistant MEL624 cells (Figure 4B). Probing nuclear lysates of low-dose PLX4720-treated cells, instead of whole-cell lysates, demonstrated substantial increases in the levels of ATF4 and CHOP within 4-6 hours. This upregulation of ER 
stress markers persisted for 48 hours at levels similar to or higher than those of the canonical ER stress activator thapsigargin. Higher doses of PLX4720 $(10 \mu \mathrm{M})$ produced even higher levels of phospho-eIF $2 \alpha$ and ATF4 at earlier time points (1-4 hours) (Supplemental Figure 4B). In support of the dose-dependent increase in PERK-related signaling associated with BRAFi, mRNA levels of activating transcription factor 3 (ATF3), which is known to coordinate transcription downstream of eIF $2 \alpha$ and in conjunction with ATF4 (27), also increased in response to PLX4720 (Supplemental Figure 4C). Other arms of the ER stress response were activated to a lesser degree in a dose- and time-dependent fashion after PLX4720 treatment, including spliced and translationally active X-box binding protein 1 (XBP1) mRNA (Supplemental Figure 4C), which is predominantly downstream of IRE1 $\alpha$ signaling.

To determine the proximal link between BRAFi and ER stress activation, a proximity ligation assay (PLA) (28) was performed on MEL624 cells using antibodies against GRP78 and BRAF, while simultaneously outlining the ER via immunofluorescence against the ER resident protein disulfide isomerase (PDI) (Figure 4C). Positive and negative PLA controls performed as expected (Supplemental Figure 5A). In the absence of BRAFi, a faint PLA signal indicated that mutant BRAF does bind to GRP78 to some degree in the absence of drug treatment. This binding occurs mainly in the cytoplasmic pool of mutant BRAF, as images of GRP78-BRAF and the ER marker did not markedly colocalize. While the GRP78 protein predominantly resides in the ER, there is a cytoplasmic variant, GRP78va, which lacks the ER localization signal but retains its cytoplasmic chaperone function (29). Within 30 minutes of treatment with PLX4720, the GRP78-BRAF PLA signal increased more than 2-fold. A substantial increase in binding between mutant BRAF within the ER pool and GRP78 was also observed. These findings suggested that while mutant BRAF bound to GRP78va constitutively, BRAFi promoted further binding of mutant BRAF and GRP78 within the ER, thereby allowing the ensuing ER stress response. To further confirm that GRP78 does in fact bind to BRAF in a drug-dependent manner, MEL624 cells were transduced with viral particles containing empty expression vector or a vector expressing Flag-tagged BRAF ${ }^{\mathrm{V} 600 \mathrm{E}}$. While total levels of GRP78 were similar in vehicle-treated vector control, vehicle-treated FlagBRAF $^{\mathrm{V} 600 \mathrm{E}}$, and PLX4720-treated Flag-BRAFV600E MEL62 4 cells, co-IP of mutant BRAF followed by immunoblotting for GRP78 revealed that significant levels of GRP78 bound to mutant BRAF in the presence, but not the absence, of the BRAF inhibitor (Supplemental Figure 5B). At the same time that PLX4720-induced binding of ER-resident mutant BRAF and GRP78 was observed, dissociation of ER-resident GRP78 and PERK was also observed (Figure 4C). Thus, the ER stress response appears to be activated in a canonical fashion in BRAF mutant melanoma cells treated with BRAF inhibitor. Drug-induced binding of GRP78 to mutant BRAF and simultaneous dissociation of this gatekeeper chaperone from the activators of the ER stress response (30) leads to activation of the ER stress response after BRAFi. In addition, a significant expansion of the ER area per cell was observed (Figure 4C and Supplemental Figure 5C). Morphological changes in the ER, consistent with ER stress, were observable by electron microscopy at later time points (Supplemental Figure 6). PLX4720 treatment produced dilated and disorganized ER with granular contents suggestive of retained unfolded proteins, similar to the ER stress inducer thapsigargin, providing morphological evidence that BRAFi induces early ER stress.
Blockade of PERK-dependent ER stress response limits BRAFi-induced autophagy and enhances cell death. To determine whether this ER stress response contributes to BRAFi-induced cell death, or rather mitigates it (like autophagy), we studied the effects of PERK kinase activity blockade, with or without BRAFi, on cell death markers using the potent and specific PERK inhibitor GSK2606414 (31). MEL624 and A375P cells treated with PLX4720 demonstrated increased levels of phospho-PERK that were nearly completely abrogated when PLX4720 was combined with GSK2606414 (Figure 5, A and B). Neither PLX4720 nor GSK2606414 treatment alone produced increased levels of the apoptosis markers cleaved PARP or cleaved caspase-3 compared with control cells; however, significant accumulation of both were observed with combined BRAF and PERK inhibition. This increased apoptosis with combined BRAFi and ER stress inhibition was further confirmed by measuring cell viability in MEL624 cells treated with DMSO, $1 \mu \mathrm{M}$ PLX4720, $1 \mu \mathrm{M}$ GSK2606414, or the combination. At both 24 and 48 hours, combined BRAFi and PERK inhibition produced additive cytotoxicity compared with either treatment alone (Figure 5C). While PERK inhibition alone had minimal effects on autophagy, as measured by LC3II/LC3I ratio, combining PERK inhibition with BRAFi completely abrogated BRAFi-induced autophagy (Figure 5D). To further confirm that the PERK arm of the ER stress response is essential for BRAFi-induced autophagy, MEL624 and A375P cells transfected with siRNA against PERK were treated with DMSO or PLX4720 (Figure 5, E and F). Knockdown of PERK was robust (Supplemental Figure 7) with separate duplexes. PLX4720 treatment resulted in a significant increase in the LC3II/ LC3I ratio in cells transfected with the siRNA against nontarget control, reflective of PLX4720-induced autophagy. In contrast, there was no PLX4720-associated increase in LC3II/LC3I ratio in cells transfected with siPERK in either cell line (Figure 5, E and F). These results establish a significant role for PERK-dependent ER stress response in PLX4720-induced autophagy.

Lysosomal autophagy inhibition angments the efficacy of BRAFi in vivo. Since autophagy inhibition with HCQ is already being tested in clinical trials, we sought to determine the effects of combining BRAFi and lysosomal autophagy inhibition in vivo. MEL624 was chosen for in vivo studies because it is naturally occurring in the $B R A F$ mutant PTEN wild-type cell line (32) that has de novo resistance to BRAFi, reflecting a large subset of BRAF mutant melanoma that may not be effectively addressed by BRAF and PI3K pathway inhibitor combinations that are entering clinical trials. Xenografts were generated in the flanks of nude mice using BRAFiresistant MEL624 cells. Mice were treated daily with either PBS or $40 \mathrm{mg} / \mathrm{kg}$ Lys05 i.p. and fed either control or PLX4720-infused chow. After 15 days of daily treatment, the Lys05 dose was reduced to $20 \mathrm{mg} / \mathrm{kg}$ to avoid bowel toxicity (20). 1 mouse treated with Lys05 died, presumably of bowel toxicity. All other mice survived the planned 22 days of treatment without significant toxicity. At the end of 22 days, the combination of Lys05 and PLX4720-infused chow was the only treatment that produced significant tumor regression compared with control (Figure 6A and Supplemental Figure 8). Cell lysates from tumors harvested at the end of this treatment were subjected to immunoblotting against phosphoMAPK, LC3, p62, and cleaved caspase-3 (Figure 6B). Quantification of Western blot bands by cohort indicated that all treatments produced reduced phospho-MAPK levels compared with control, even after 22 days of treatment. BRAFi alone significantly decreased p62, and BRAFi combined with Lys05 led to slightly increased p62 lev- 
els, as expected, due to simultaneous autophagy induction and distal blockade. PLX4720 treatment, both alone and in combination with Lys05, produced an increase in LC3II/LC3I ratio compared with controls. Cleaved caspase-3 was only evident in tumor lysates from mice treated with combined PLX 4720 and Lys05, providing in vivo evidence that this combination enhances cell death compared with single-agent therapy. Electron microscopy demonstrated evident AV accumulation in tumors harvested from mice treated with single-agent Lys05- or PLX4720 compared with PBS treatment (Figure 6C). Massive AV accumulation was observed in tumors in mice treated with combined PLX4720 and Lys05.

\section{Discussion}

Fluoro-deoxyglucose PET scan studies have demonstrated that BRAFi produces rapid, strikingly homogenous, and profound metabolic effects to all tumors in most BRAF mutant melanoma patients (33). This finding, and the significant survival benefit of single-agent vemurafenib, firmly established oncogenic BRAF as a driver mutation. BRAF mutant melanoma cells are therefore addicted to signaling through mutant BRAF. However, while some patients experience a prolonged tumor response that translates into a significant survival benefit (2), other patients with larger, bulkier tumors are likely to have short-lived tumor responses and progress rapidly (34). There have been a number of escape mechanisms described for BRAF mutant melanomas, including mutations and posttranslational alterations that activate either the MAPK pathway $(16,35)$ or the PI3K signaling pathway $(15)$ in the face of continued BRAFi. A splicing variant of mutated BRAF was described as the first example of an alteration directly in the BRAF protein conformation that allows BRAF mutant melanoma cells to escape BRAFi-induced cell death (36). Some of these cell-intrinsic mechanisms of resistance are thought to be present in a small subset of cancer cells within tumors prior to starting treatment, rather than being directly induced by BRAFi.

More recently, numerous adaptive mechanisms of resistance have been described, whereby BRAFi induces a resistance mechanism in a more substantial population of cells. For instance, FOXD3-dependent ERBB3 activation has been found to promote BRAFi resistance (37). Upregulation of antiapoptotic BCL2A1 (38) and reprogramming of mitochondrial metabolism through MITF-dependent transcription (39) have also has been implicated as resistance mechanisms. The tumor microenvironment can also promote BRAFi resistance through stromal cell secretion of hepatocyte growth factor and engagement of MET signaling in BRAF mutant melanoma cells (40). The homogenous metabolic compromise to cancer cells, and the heterogeneous mechanisms of resistance and clinical outcome that result in patients, prompted us to study the effect of cytoprotective stress responses downstream of perturbed growth factor signaling, and lead us to the discovery of BRAFi-induced autophagy as an adaptive resistance mechanism to BRAFi. Unlike intrinsic resistance mechanisms (such as an oncogene mutation present in pretreatment tumor samples that confers immediate resistance to a subpopulation of tumors cells) or acquired resistance mechanisms (where reliance on alternative signaling pathways emerges over time, specifically in the resistant cell population), adaptive resistance mechanisms are not turned on to a substantial degree in cells before treatment, but rather are hardwired responses to drugs that occur early in the course of therapy, both in cells that are destined to die (sensitive) and in cells that are destined to survive therapy (resistant). Our data suggest that in certain model contexts, cells that have either intrinsic or acquired BRAFi resistance can also rely on BRAFi-induced autophagy more heavily for survival than BRAFi-sensitive cells. Importantly, blockade of autophagy did not antagonize BRAFi-induced cell death in BRAFi-sensitive cells, and in certain long-term cell culture models, this approach demonstrated additive cytotoxicity as well. Maximizing cell killing by initially targeting mutant BRAF and components of stress responses, such as autophagy, could prevent the emergence of cells that already have resistance to BRAFi due to bypasses in MAPK or alternative signaling pathways.

Autophagy occurs at basal levels in virtually all cells, but is upregulated when cells face metabolic or therapeutic stress. During tumor response, in patients treated with vemurafenib, autophagy is increased in the surviving cells that are destined to recur. Our in vitro data, which demonstrated that autophagy was induced in both BRAFi-sensitive and BRAFi-resistant cell lines, indicate that cells that are dying or dead in response to BRAFi may have also induced autophagy as an attempt to survive the stress, but autophagy was inadequate to stop death due to BRAFi in sensitive cells. Consequently, combining autophagy blockade with BRAFi led to augmented cell death and growth impairment compared with BRAFi alone in both $2 \mathrm{D}$ and $3 \mathrm{D}$ culture. The results of the current study support therapeutic targeting of autophagy in melanoma and provide the preclinical rationale for launching clinical trials of the combination of BRAF and autophagy inhibitors.

The identification of the ER stress response as the mechanistic link between BRAF signaling and cytoprotective autophagy establishes a new signaling axis that has multiple druggable targets. Our finding that mutant BRAF bound GRP78 in the presence of BRAFi identifies the proximal molecular link between MAPK signaling and the ER stress response in BRAF mutant melanoma cells. BRAFi-induced binding of mutant BRAF to the ER stress gatekeeper GRP78 within the ER is a new and unexpected molecular interaction. While BRAF lacks the predicted signal peptide, or the ER retention peptide KDEL, it may be that in the presence of BRAFi, a pool of mutant BRAF gets redirected or trapped in an unfolded state in the ER during translation. Further study is needed to confirm this subcellular localization, understand how mutant BRAF enters the ER, and determine the functional role (if any) for mutant BRAF within the ER.

Previous work in yeast (41) and mammalian cells $(42,43)$ has established that ER stress can activate autophagy. We have previously found that the stress of forced Myc expression activates an ER stress response, followed by PERK-mediated processing of LC3 and increased autophagic flux (44). The PERK-eIF2 $\alpha$-ATF4 axis is likely the critical connection between BRAF signaling and autophagy, since phosphorylation of eIF $2 \alpha$ inhibited global translation accompanied with selective translation of ATF4, a transcription factor that regulates LC3 expression. ATF4 has been shown by at least 2 groups to stimulate autophagy $(45,46)$. The contribution of IRE $1 \alpha$ - and ATF $6 \alpha$-dependent ER stress response signaling to activation of autophagy has been less well studied. In IRE1 $\alpha$ dependent signaling, JNK is a known regulator of autophagy, both at the transcriptional level and through the phosphorylation of BCL2 and displacement of Beclin-BCL2 binding (47). The influence of the transcription factors XBP1 and CHOP and ATF $6 \alpha$ on the activation of cytoprotective autophagy is unknown.

A previous report found that the ER stress response that ensues after BRAFi in some BRAF mutant cell lines may contribute to BRAFi-associated apoptosis (48). In our present work, chemical 
and siRNA studies demonstrated that inhibition of components of the PERK arm of the ER stress response blunted PLX4720-induced autophagy and enhanced cell death. While it is clear that activation of an uncontrolled ER stress response or autophagy can contribute to cell death (26), our data support that in the setting of BRAFi, ER stress-induced autophagy can promote cell survival (Supplemental Figure 9). While there are currently no clinically available inhibitors that target components of the ER stress response or proximal autophagy inhibitors available to test in combination with BRAF inhibitors, the distal lysosomal autophagy inhibitor HCQ is being tested in multiple combination regimens for melanoma and other diseases. Based on the results of the present work, a clinical trial testing the safety of the combination of vemurafenib and HCQ in patients with BRAF mutant melanoma has already been launched (ClinicalTrials.gov ID NCT01897116).

\section{Methods}

Patient samples and IHC. Patients' BRAF mutation status was confirmed by DNA sequencing (49). For LC3 IHC studies, FFPE slides were made from either freshly obtained biopsy tissue or, if unavailable, archival tissue. Slides were stained with anti-LC3B (Abcam Inc.; 1:500, with citric acid/ antigen retrieval) by standard immunoperoxidase techniques all together by the Fontana-Masson procedure, which differentiates LC3B-positive tumor cells (brown) from tumor-associated melanophages (azure) (50). Original images were produced with a Zeiss Axioskop 40 light microscope equipped with a Spot Flex digital camera. All images were cut and assembled together into a single Photoshop document, merged into a single layer, and then treated with automatic contrast and brightening tools. LC3B staining intensity was assessed by 2 trained observers blinded to treatment, with independent agreement in 12 of 14 pairs (87\%). Scoring was by consensus for the remaining 2 pairs. Scoring was as follows: 1, weak; 2 , moderate; 3 , strong; 4 , very strong.

Cell culture and reagents. Melanoma cell lines A375P, SKMEL5, 1205Lu, MEL624, MEL1617, MEL1617R, WM983B, WM983BR, and A375P mCherryGFP-LC3 were maintained in RPMI-1640 (Invitrogen) supplemented with $10 \%$ fetal bovine serum (Sigma-Aldrich), $50 \mu \mathrm{g} / \mathrm{ml}$ gentamicin, and 25 $\mathrm{mmol} / \mathrm{l} \mathrm{HEPES}$ in the presence of $5 \% \mathrm{CO}_{2}$ at $37^{\circ} \mathrm{C}$. The BRAFi-resistant cell lines WM983BR and MEL1617R were generated and maintained as previously described (15). PLX4720 was provided by Plexxikon. Rapamycin and thapsigargin were purchased from Sigma-Aldrich. HCQ was purchased from Spectrum Chemicals. GSK2118436 and GSK1120212 were purchased from Selleck Chemicals. GSK2606414 was provided by Glaxo Smith Kline. PLX4720 and PLX4720-infused chow was provided by Plexxicon.

Electron microscopy. Electron microscopy was performed as previously described (12).

Immunoblotting and IP. Immunoblotting was performed on whole-cell lysates (12) and nuclear extracts as previously described (44). Cell Signaling Technology antibodies used were as follows: phospho-AMPK $\alpha$ (Thr172), AMPK $\alpha$ (23A3), phospho-ULK1 (Ser757), ULK1 (A705), cleaved caspase-3 (Asp175), phospho-eIF2 $\alpha$ (Ser51), eIF2 $\alpha$, PERK (C33E10), phospho-p70S6K (Thr389), and p70S6K. Santa Cruz antibodies used were as follows: CHOP (F-168), CHOP/GADD 153 antibody (B-3), p62/SQSTM1 (D-3), actin (I-19), ATF4/CREB-2 (C-20), and Raf-B (C-19). Novus Biologicals antibody used were as follows: ATG5 ([DyLight 488]). A rabbit polyclonal antibody against LC3 was generated from QCB biological using rat LC3B N terminus peptide as previously described (51). Phospho-PERK was a gift from A. Diehl (University of Pennsylvania, Philadelphia, Pennsylvania, USA). Co-IP was performed using Pierce Crosslink Kit (Thermo Scientific; catalog no. 26417). Briefly, MEL624 cells were transduced with viral particles of Flag-tagged BRAF ${ }^{\mathrm{V} 600 \mathrm{E}}$ or empty vector, then selected with $10 \mu \mathrm{g} / \mathrm{ml}$ blasticidin for 72 hours. After blasticidin withdrawal for 24 hours, cells were treated with DMSO or $10 \mu \mathrm{M}$ PLX4720 for 30 minutes, then harvested for Co-IP. Anti-BRAF polyclonal antibody (10 $\mu \mathrm{g}$; Santa Cruz Biotechnology, SC-166) was coupled to $20 \mu \mathrm{l}$ Pierce Protein A/G Plus Agarose into a Pierce Spin Column, and Pierce Control Agarose Resin was used as a negative control. Co-IP reactions were performed at $4{ }^{\circ} \mathrm{C}$ overnight. Eluates were resolved by SDS-PAGE and analyzed by Western blot for GRP78 (GRP78 antibody; Santa Cruz Biotechnology, SC-1051) and for Flag-BRAF ${ }^{\mathrm{V} 600 \mathrm{E}}$ using both anti-FLAG M2 (Sigma-Aldrich, F3165) and anti-BRAF antibody (Santa Cruz Biotechnology, SC-166). Densities from Western blots were quantified using Adobe Photoshop CS4 Extended.

PLA. PLA was performed using Duolink In Situ fluorescence technology (Olink Bioscience). Briefly, MEL624 cells were plated into 16-well Chamber Slides (Lab-Tek) and grown overnight. The cells were fixed in $4 \%$ formaldehyde for 15 minutes and permeabilized with $0.2 \%$ Triton X-100 in PBS for 10 minutes. Cells were blocked with $1 \%$ BSA for 40 minutes, then incubated with combinations of goat anti-GRP78 polyclonal antibody (Santa Cruz, sc-1051, sc-1050), rabbit anti-BRAF polyclonal antibody (Santa Cruz, sc-166) and rabbit anti-PERK polyclonal antibody (Santa Cruz, sc-13073) overnight at $4{ }^{\circ} \mathrm{C}$. The primary antibodies were detected with secondary antibodies conjugated with the oligonucleotides of Anti-Goat PLUS (Sigma-Aldrich, DUO92003) and Anti-Rabbit MINUS (Sigma-Aldrich, DUO92005) at 1:5 dilution in 1\% BSA buffer for 1 hour. The PLA reaction of goat anti-GRP78 and rabbit IgG isotype (Novus, NB600-441) with AntiGoat PLUS/Anti-Rabbit MINUS was used as a technical negative control, and goat anti-GRP78 and rabbit anti-ATM (Santa Cruz, sc-7230) with Anti-Goat PLUS/Anti-Rabbit MINUS was used as a biological negative control. Detection of BRAF using rabbit anti-BRAF with Anti-Rabbit PLUS (Sigma-Aldrich, DUO92002) and Anti-Rabbit MINUS was used as a technical positive control. Ligation and amplification steps were performed using Duolink In Situ Detection Reagents Red (Sigma-Aldrich, DUO92008) according to the manufacturer's instructions. To detect ER, the SelectivFX Fluor 488 ER Labeling Kit (Invitrogen, S34253) was used with a modified procedure and incorporated into the above PLA assay. Images were acquired on a Zeiss Axioplan Microscope at $\times 20, \times 40$, and $\times 100$ magnification. Quantification of intensity and area of fluorescent signal was performed by applying the Adobe Photoshop magic wand tool to specific colors on unadjusted TIFF images collected directly from the microscope.

MTT assay, clonogenic assay, and $3 D$ culture. For the MTT assay, cells were treated in 6 replicates for 72 hours. MTT reagent (Roche) was applied, cells were solubilized, and absorbance was read at $570 \mathrm{~nm}$, with background subtraction at $690 \mathrm{~nm}$. The clonogenic assay was performed as previously described (52). Briefly, cells were suspended in RPMI-1640 containing $10 \%$ FBS, then plated in 6-well plates $\left(1 \times 10^{3}\right.$ cells/well). Medium was changed and drug treated every 3-4 days. After incubation for 2 weeks, colonies were stained with crystal violet and counted. 3D spheroid experiments were conducted as previously described (12) using the Live/Dead Assay (Molecular Probes). Spheroid dimensions and quantification of percentage of dead cells was done using the lasso and magic wand tools in Adobe Photoshop Extended CS4.

RNA interference. Knockdown of human PERK expression was performed using Thermo Scientific Dharmacon ON-TARGETplus siRNA (catalog nos. NT D-001810-10-05, J-004883-09, and J-004883-10) and Lipofectamine RNAiMAX (Invitrogen). Briefly, MEL624 and A375P cells were plated at a density of $30 \%$ confluence at the time of transfection. After 48 hours, cells were treated with DMSO or PLX4720 and cultured for 24 hours, and protein expression levels were then measured by Western blotting. Knockdown of ATG5 expression was performed using the following Sigma-Aldrich mission shRNA vectors: TRCN0000150940, 0000151963, 0000150645, 0000150976, and 0000151474. TRC Non-Target shRNA vec- 
torSHC002 was used as negative control. Cells were transfected with the above vectors using Lipofectamine 2000 reagent (Invitrogen) and selected with puromycin. Knockdown of BRAF was accomplished using OpenBiosystems shRNA pLKO.1 plasmids. Specifically, MEL624 and A375P cells were transduced with the lentiviral particles, which were produced by cotransfection of shBRAF (hp3, TRCN0000006293, sequence TTGCTGGTGTATTCTTCATAG; hp4, TRCN0000006292, sequence TTTGAAGGCTTGTAACTGCTG) and shNT (sequence CCTAAGGTTAAGTCGCCCTCGCTCGAGCGAGGGCGACTTAACCTTAGG) with lentiviral packaging plasmid mix into the packaging cell line HEK293. The transduced MEL624 and $\mathrm{A} 375 \mathrm{P}$ cells were selected by puromycin until cells were to be assayed, and then protein expression levels were measured by Western blotting.

Tumor xenograft experiments. All experiments were carried out using 5-weekold $n u / n u$ nude mice (Charles River Labs). Tumor generation, tumor measurements, and tissue harvesting were performed as previously described (20).

Statistics. To assess statistical significance in experiments directly comparing an experimental group with a control group, Student's unpaired, 2 -tailed $t$ test was used. A $P$ value less than 0.05 was considered significantly different from the null hypothesis. Kaplan-Meier survival analysis was conducted using Graphpad Prism, and the log-rank test was used to assess statistical significance of differences between survival curves.
Study approval. For patient materials, informed consent on a University of Pennsylvania IRB-approved tissue collection protocol was obtained for each patient prior to biopsy or retrieval of archival tumor tissue and correlation with clinical outcome. Approval for animal care and use for these experiments was provided by the University of Pennsylvania IACUC.

\section{Acknowledgments}

We thank Adina Monica-Vultur for guidance on 3D culture and Ray Meade (Biomedical Imaging Core, Abramson Cancer Center) for outstanding preparation of electron microscopy specimens. This work was supported by NIH grants K23CA120862 and P01CA114046 (to R.K. Amaravadi) and 1R01 CA139362 (to C. Koumenis).

Received for publication July 27, 2013, and accepted in revised form November 22, 2013.

Address correspondence to: Ravi Amaravadi, Department of Medicine and Abramson Cancer Center Perelman School of Medicine, University of Pennsylvania, 16 Penn Tower, 3400 Spruce Street, Philadelphia, Pennsylvania 19104, USA. Phone: 215.662.7402; Fax: 215.349.8550; E-mail: Ravi.amaravadi@uphs.upenn.edu.
1. Flaherty KT, et al. Inhibition of mutated, activated BRAF in metastatic melanoma. $N$ Engl J Med. 2010;363(9):809-819.

2. Sosman JA, et al. Survival in BRAF V600-mutant advanced melanoma treated with vemurafenib. NEngl J Med. 2012;366(8):707-714.

3. Hauschild A, et al. Kaempgen E, et al. Dabrafenib in BRAF-mutated metastatic melanoma: a multicentre, open-label, phase 3 randomised controlled trial. Lancet. 2012;380(9839):358-365.

4. Villanueva J, Vultur A, Herlyn M. Resistance to BRAF inhibitors: unraveling mechanisms and future treatment options. Cancer Res. 2011;71(23):7137-7140.

5. Amaravadi RK, et al. Principles and current strategies for targeting autophagy for cancer treatment. Clin Cancer Res. 2011;17(4):654-666.

6. Amaravadi RK, Thompson CB. The roles of therapy-induced autophagy and necrosis in cancer treatment. Clin Cancer Res. 2007;13(24):7271-7279.

7. Fan QW, et al. Akt and autophagy cooperate to promote survival of drug-resistant glioma. Sci Signal. 2010;3(147):ra81.

8. Amaravadi RK, et al. Autophagy inhibition enhances therapy-induced apoptosis in a Myc-induced model of lymphoma. J Clin Invest. 2007;117(2):326-336.

9. Kanzawa T, Germano IM, Komata T, Ito H, Kondo Y, Kondo S. Role of autophagy in temozolomideinduced cytotoxicity for malignant glioma cells Cell Death Differ. 2004;11(4):448-457.

10. Degtyarev M, et al. Akt inhibition promotes autophagy and sensitizes PTEN-null tumors to lysosomotropic agents. J Cell Biol. 2008;183(1):101-116.

11. Rosenfeld MR GS, et al. Pharmacokinetic analysis pharmacodynamic evidence of autophagy inhibition in patients with newly diagnosed glioblastoma treated on a phase I trial of hydroxychloroquine in combination with adjuvant temozolomide radiation (ABTC 0603). JClin Oncol. 2010; 28(15 suppl):3086.

12. Ma XH, et al. Measurements of tumor cell autophagy predict invasiveness, resistance to chemotherapy, and survival in melanoma. Clin Cancer Res. 2011;17(10):3478-3489.

13. Lazova R, Klump V, Pawelek J. Autophagy in cutaneous malignant melanoma. J Cutan Pathol. 2010;37(2):256-268.

14. Lazova R, Camp RL, Klump V, Siddiqui SF, Amaravadi RK, Pawelek JM. Punctate LC3B expression is a common feature of solid tumors and associated with proliferation, metastasis, and poor outcome.
Clin Cancer Res. 2012;18(2):370-379.

15. Villanueva J, et al. Acquired resistance to BRAF inhibitors mediated by a RAF kinase switch in melanoma can be overcome by cotargeting MEK and IGF-1R/PI3K. Cancer Cell. 2010;18(6):683-695.

16. Nazarian R, et al. Melanomas acquire resistance to B-RAF(V600E) inhibition by RTK or N-RAS upregulation. Nature. 2010;468(7326):973-977.

17. Tanida I, Ueno T, Kominami E. LC3 conjugation system in mammalian autophagy. Int J Biochem Cell Biol. 2004;36(12):2503-2518.

18 . Klionsky DJ, et al. Guidelines for the use and interpretation of assays for monitoring autophagy. Autophagy. 2012;8(4):445-544.

19. Flaherty KT, et al. Combined BRAF and MEK inhibition in melanoma with BRAF V600 mutations. NEngl J Med. 2012;367(18):1694-1703.

20. McAfee Q, et al. Autophagy inhibitor Lys05 has single-agent antitumor activity and reproduces the phenotype of a genetic autophagy deficiency. Proc Natl Acad Sci U S A. 2012;109(21):8253-8258.

21. Zheng B, et al. Oncogenic B-RAF negatively regulates the tumor suppressor LKB1 to promote melanoma cell proliferation. Mol Cell. 2009;33(2):237-247.

22. Esteve-Puig R, Canals F, Colome N, Merlino G, Recio JA. Uncoupling of the LKB1-AMPKalpha energy sensor pathway by growth factors and oncogenic BRAF. PLoS One. 2009;4(3):e4771.

23. Egan DF, et al. Phosphorylation of ULK1 (hATG1) by AMP-activated protein kinase connects energy sensing to mitophagy. Science. 2011;331(6016):456-461.

24. Jung CH, et al. ULK-Atg13-FIP200 complexes mediate mTOR signaling to the autophagy machinery. Mol Biol Cell. 2009;20(7):1992-2003.

25. Kim I, Xu W, Reed JC. Cell death and endoplasmic reticulum stress: disease relevance and therapeutic opportunities. Nat Rev Drug Discov. 2008;7(12):1013-1030.

26. Walter P, Ron D. The unfolded protein response: from stress pathway to homeostatic regulation. Science. 2011;334(6059):1081-1086.

27. Jiang HY, et al. Activating transcription factor 3 is integral to the eukaryotic initiation factor 2 kinase stress response. Mol Cell Biol. 2004;24(3):1365-1377.

28. Soderberg O, et al. Direct observation of individual endogenous protein complexes in situ by proximity ligation. Nat Meth. 2006;3(12):995-1000.

29. Ni M, Zhang Y, Lee AS. Beyond the endoplasmic reticulum: atypical GRP78 in cell viability, signalling and therapeutic targeting. The Biochem J.
2011;434(2):181-188

30. Bertolotti A, Zhang Y, Hendershot LM, Harding HP, Ron D. Dynamic interaction of BiP and ER stress transducers in the unfolded-protein response. Nat Cell Biol. 2000;2(6):326-332.

31. Axten JM, et al. Discovery of 7-methyl-5-(1-\{[3(trifluoromethyl)phenyl]acetyl\}-2,3-dihydro-1Hindol-5-yl)-7H-pyrrolo[2,3-d]pyrimidin-4-amine (GSK2606414), a potent and selective first-in-class inhibitor of protein kinase R (PKR)-like endoplasmic reticulum kinase (PERK). J Med Chem. 2012;55(16):7193-7207.

32. Deng W, Gopal YN, Scott A, Chen G, Woodman SE, Davies MA. Role and therapeutic potential of PI3K-mTOR signaling in de novo resistance to BRAF inhibition. Pigment Cell Melanoma Res. 2012;25(2):248-258.

33. McArthur GA, et al. Marked, homogeneous and early FDG-PET responses to vemurafenib in BRAF-mutant advanced melanoma. J Clin Oncol. 2012;30(14):1628-1634

34. Amaravadi RK, et al. Prolonged responses to vemurafenib in patients with BRAFV600 mutant melanoma with low tumor burden at baseline. Poster presented at: Society for Melanoma Research annual meeting; November 9-13, 2011; Tampa, Florida, USA. Poster \#3.

35. Johannessen CM, et al. COT drives resistance to RAF inhibition through MAP kinase pathway reactivation. Nature. 2010;468(7326):968-972.

36. Poulikakos PI, et al. RAF inhibitor resistance is mediated by dimerization of aberrantly spliced BRAF(V600E). Nature. 2011;480(7377):387-390.

37. Abel EV, et al. Melanoma adapts to RAF/MEK inhibitors through FOXD3-mediated upregulation of ERBB3. J Clin Invest. 2013;123(5):2155-2168.

38. Haq R, et al. BCL2A1 is a lineage-specific antiapoptotic melanoma oncogene that confers resistance to BRAF inhibition. Proc Natl Acad Sci U S A. 2013;110(11):4321-4326.

39. Haq R, et al. Oncogenic BRAF regulates oxidative metabolism via PGC1 $\alpha$ and MITF. Cancer Cell. 2013;23(3):302-315.

40. Straussman R, et al. Tumour micro-environment elicits innate resistance to RAF inhibitors through HGF secretion. Nature. 2012;487(7408):500-504.

41. Bernales S, Schuck S, Walter P. ER-phagy: selective autophagy of the endoplasmic reticulum. Autophagy. 2007;3(3):285-287.

42. Yorimitsu T, Nair U, Yang Z, Klionsky DJ. Endo- 
plasmic reticulum stress triggers autophagy. J Biol Chem. 2006;281(40):30299-30304

43. Kruse KB, Brodsky JL, McCracken AA. Autophagy: an ER protein quality control process. Autophagy. 2006;2(2):135-137.

44. Hart LS, et al. ER stress-mediated autophagy promotes Myc-dependent transformation and tumor growth. J Clin Invest. 2012;122(12):4621-4634.

45. Rzymski T, et al. Regulation of autophagy by ATF4 in response to severe hypoxia. Oncogene. 2010;29(31):4424-4435.

46. Rouschop KM, et al. The unfolded protein response protects human tumor cells during hypoxia through regulation of the autophagy genes MAP1LC3B and ATG5.J Clin Invest. 2010;120(1):127-141.

47. Wei Y, Pattingre S, Sinha S, Bassik M, Levine B. JNK1-mediated phosphorylation of Bcl-2 regulates starvation-induced autophagy. Mol Cell. 2008;30(6):678-688.

48. Beck D, et al. Vemurafenib potently induces endoplasmic reticulum stress-mediated apoptosis in BRAFV600E melanoma cells. Sci Signal. 2013;6(260):ra7.

49. Chapman PB, et al. Improved survival with vemu- rafenib in melanoma with BRAF V600E mutation. N Engl J Med. 2011;364(26):2507-2516.

50. Kamino H, Tam ST. Immunoperoxidase technique modified by counterstain with azure $\mathrm{B}$ as a diagnostic aid in evaluating heavily pigmented melanocytic neoplasms. J Cutan Pathol. 1991;18(6):436-439.

51. Lum JJ, DeBerardinis RJ, Thompson CB. Autophagy in metazoans: cell survival in the land of plenty. Nat Rev Mol Cell Biol. 2005;6(6):439-448.

52. Franken NA, Rodermond HM, Stap J, Haveman J, van Bree C. Clonogenic assay of cells in vitro. Nat Protoc. 2006;1(5):2315-2319. 